\title{
TECHNOLOGY OF INTELLIGENT DISTRIBUTION TERMINAL WITH THE ABILITY OF UPDATING DISTRIBUTION CIRCUIT TOPOLOGY
}

\author{
Jun MEI ${ }^{1}$, Yong WANG ${ }^{2}$, Wenlu $\mathrm{JI}^{2}$, Ming ZHANG ${ }^{2}$, Xun XU ${ }^{1}$, Zhihua \\ Zhang ${ }^{3}$, Mingxiang $\mathrm{Liu}^{3}$ \\ ${ }^{1}$ School of Electrical Engineering, Southeast University, Nanjing 210096, Jiangsu Province, China \\ ${ }^{2}$ Nang Jing Power Supply Company, Nanjing 210019, Jiangsu Province, China \\ ${ }^{3}$ Nari Technology Development Limited Company, Nanjing 210061, Jiangsu Province, China
}

Keywords: distribution network, distribution line, topology, change, model interaction mechanism, self-adaption

Abstract. With the increasing requirement of the self-adaptation topology change of distribution line, not only should FA realize distribution automation terminal plug and play, but also be able to adapt to changes in the topology of distribution lines. Distribution circuit topology model can reflect the connection of primary equipment and the parameters of distribution operation, which plays a very important role in the safe and stable operation of the distribution network. In the actual operation of the distribution network, in addition to changes in the topology caused by a device, distribution line load transfer, fault isolation, non fault area to restore power supply will also cause topology changes. Therefore, the static topology model and dynamic topology model of distribution lines are introduced as the basis for the realization of the topology adaptation of distribution lines.

\section{Introduction}

Nowadays, With the rapid development of smart grid technology, Smart distribution network as a key link in the smart grid has got more and more attention from experts and scholars. Among them, the power distribution automation terminal technology is the research hotspots in this field. At present, the research of terminal is mainly focused on the plug and play technology[1-3], the system configuration method[4-5] and the terminal communication technology[6-8]. However, with the development of intelligent distribution network, not only should the intelligent distribution monitoring terminal monitor the operation condition of the primary equipment, but also need to judge and analyze the topology structure of distribution line. So as to increase the accuracy of feeder automation (FA) on the fault location, isolation and restoration of distribution network and improve the reliability of power supply.

According to the different working mechanism, feeder automation is divided into centralized FA and distributed FA. The centralized FA relies too much on the accuracy of the global information of the master station which will create great impact on the reliability of power supply. Distributed FA can finish fault location, isolation and restoration of distribution network through the coordination and cooperation of adjacent terminals which can achieve the rapid isolation of the fault and self-healing. In the current intelligent distributed FA application, there are a lot of problems in the maintainability and adaptability to the change of distribution line:

(1) The Self-networking capability of intelligent distributed FA is poor: The accuracy of the movement is closely related to the topology structure of distribution line. There is no method to automatically update the circuit topology model installed in distribution automation terminal when the topology of distribution line is changed. That's why the distribution automation terminal can't achieve the function of Self-networking when the distribution line topology changes.

(2) The ability of interaction between Intelligent distributed FA and distribution automation master station is poor. The information exchange and coordination between intelligent distributed FA and distribution automation master station have not been solved properly along with the application of intelligent distributed FA, Intelligent distributed FA and distribution automation main station centralized FA operate independently, Lack of coordination ability. 
Based on the intelligent distributed FA, a distribution automation terminal technology which has the ability of distribution Self-networking topology is presented in the paper. The requirement of distribution automation for circuit topology updating is analyzed. The static topology and dynamic topology of distribution lines are defined and The interaction mechanism between master station system and distributed FA is presented. in Tests were carried out in the NARI network static simulation system.

\section{Distribution line topology}

Distribution circuit topology is primary equipment on distribution lines and their connection relationship. As shown in figure 1, the line is composed of substation outlet power supplies, circuit breakers, load switches and a contact switch. The corresponding circuit topology includes the device as well as the connection relationship as shown in Fig.1

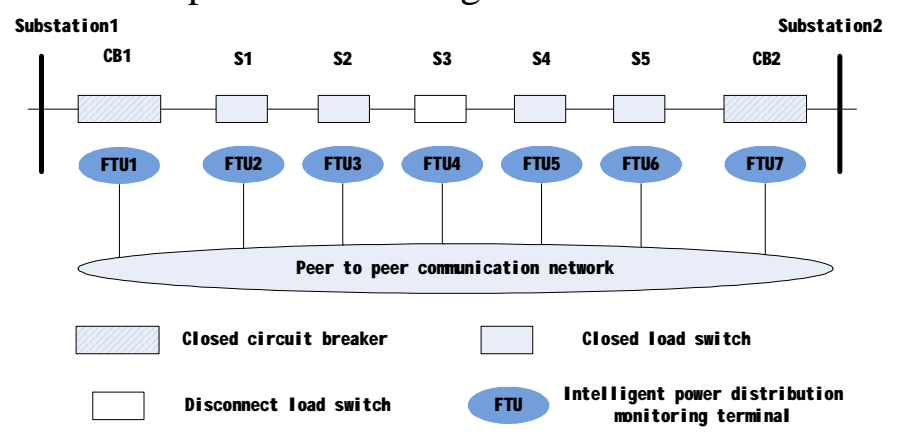

Fig. 1 The typical distribution line

In order to propose methods to update the line topology model installed in the distribution automation terminal, this document defines the distribution line topology models installed in the master system and terminal equipment. Unified Modeling based on CIM (Common Information Model) of the master system has been formed, distribution line topology CIM-based modeling approach to characterize the distribution network connection between primary devices by endpoints and connection points. For line topology model installed in the distribution terminals, the modeling methods based on IEC61850 standard is proposed in this paper. The modeling standard of topology model in substation IED (Intelligent Electronic Device) is introduced to the distribution terminal, every distribution terminal stores SCL file based on XML syntax to characterize connection between primary devices.

The main cause of the distribution line topology changes fall into two categories: One is caused by increase or decrease in distribution equipment which is called static topology change; the other topology change is due to the distribution network for load transfer, fault isolation, recovery circuit which is called dynamic topology change. Depending on the cause of the above distribution line topology change, this paper defines the distribution line static and dynamic topology.

\section{Distribution line static topology model}

Distribution line static topology model is stored in the master system. The model contains an object description of the adjacent nodes with a direct electrical connection. CIM based modeling method and XML syntax are used in the modeling. The static topology model is expressed by endpoints, connection points, and connections between primary devices. It is only needed to modify the connection relation of the adjacent nodes to realize the static topology adaptation of distribution lines when the distribution line static topology changes.

The object description of the adjacent nodes with a direct electrical connection can meet adaptive requirements of different network structures, including ring, hand in hand, petal shaped and so on.

\section{Distribution line dynamic topology model}

In the distribution network, the changes of node power supply, power supply path and the corresponding contact switch can affect operation mode of a node. The dynamic topology operation model of the power distribution line is built up according to those important characteristics, combined with the static topology information model of distribution line. 
The dynamic topology model of distribution line is not only stored in the master system, and each distribution terminal storages its related line dynamic topology model which is also called the line topology operation model. Terminal model is based on the XML syntax of the SCL file, the designed model mapping device is used to complete the conversion of two kinds of files between the master system and the terminal. Taking figure 1 for an example, the dynamic topology of FTU1 includes the connection relationship of CB1, Switch status: closed, Power supply: Substation1, Power supply path: from Substation1 to CB1, Contact switch: S3; the dynamic topology of FTU4 includes the connection relationship of S3,Switch status: disconnect, Power supply: 0, Power supply path: 0, Contact switch:0. The dynamic topology model of distribution line reflects the power supply path of feeder switching state and power supply.

Dynamic topology model of distribution line is the model base of distributed FA.

\section{The model interaction mechanism of distribution line topology}

In order to ensure the correctness of the topology information, the interaction mechanism of the terminal topology operation model must be able to respond to the state change of the distribution line switch in real time. At the same time, in order to further ensure the accuracy of the model and the reliability of the distributed FA function, we establish a reasonable system of the master station and the topology model of distributed FA, so that the terminal can update the topology operation model in real time and play a backup role when the distributed FA is in trouble.

\section{Interaction mechanism between master station system and distributed FA topology model}

As shown in Figure 2, the interaction mechanism between the master system and the distributed FA topology model is shown. First of all, we relay a wiring diagram of power distribution lines obtained from the distribution planning department to generate the distribution line static topology through the main station system feeder configuration tool. According to the definition of the dynamic topology, the main station receives the real-time information (switching state, power supply, etc.), and the dynamic topology of the distribution line is generated. Then, the dynamic topology is transformed into the operational topology which can be identified by the model. Finally, the operation topology is sent to the corresponding distribution automation terminal.

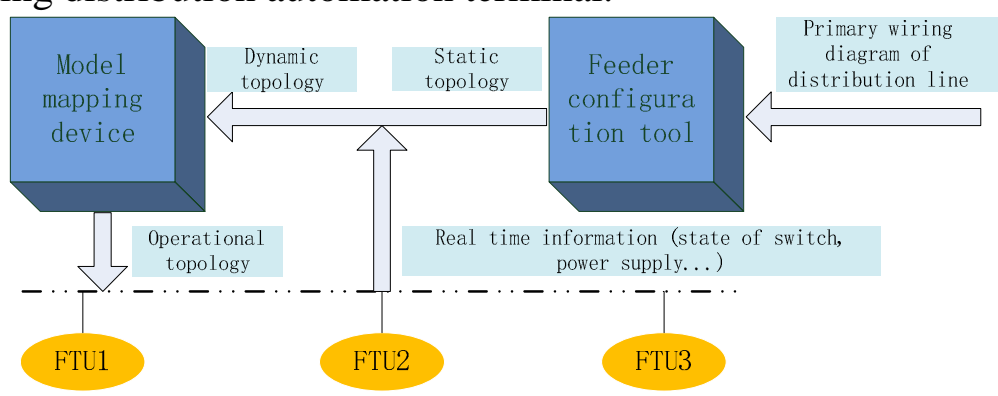

Fig .2 Interaction mechanism between master station and distributed FA

The interaction mechanism between the master station system and the distributed FA topology model enables the intelligent terminal to receive the topology of distribution line in real time, which lays the foundation for the realization of the distribution line topology adaptation.

Interaction mechanism of topology operation model between terminals

The connection of the monitoring equipment is reflected by the operation topology of the intelligent distribution terminal. Connected device and adjacent terminal can be identified by the connection relationship. the adjacent terminals can establish a communication connection through the IP address, so that the interaction of the topology operation model between terminals can be completed.

\section{Processing scheme for topology change of distribution line}

In the actual operation of the distribution network, the topology changes of distribution lines occur frequently, which has a great impact on the operation of the distributed FA. In literature, a 
method is proposed to automatically update the topology of distribution line by step by step query through the adjacent intelligent distribution terminals which can quickly identify the topology changes, but the proposed scheme does not have backup protection function of the main station system. The distribution network fault processing mode of centralized intelligence and distributed intelligent coordination is introduced in literature. The coordination mechanism of master station system and distributed FA is introduced. However, the proposed scheme does not have the function of updating the topology of distribution lines.

Based on the proposed scheme of the above documents, according to the different causes of distribution line topology change, the proposed scheme can meet the requirement of topology change adaptation, the coordination and cooperation between the main station system and intelligent distribution terminal is taken into account.

\section{Processing scheme for static topology change of distribution line}

When the distribution line static topology changes, the static topology of the main station is modified by the GIS system which is connected with the master station system, and then the dynamic topology is updated. The master station system will interact with the distribution automation terminal and make topological judgment, so as to realize the static topology adaptation of distribution line. The specific adaptive process is shown in Figure 3.

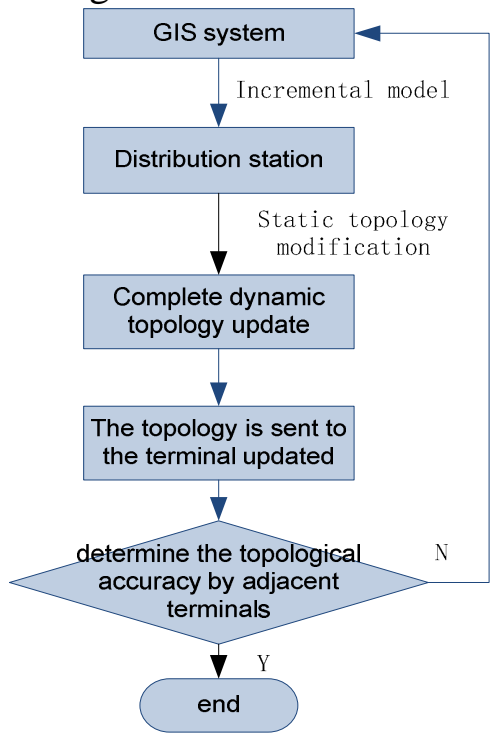

Fig .3 Static topology adaptation of power distribution lines

Step 1:GIS system promote the main station to carry out static topology modification through incremental model;

Step 2: master station complete the static topology update according to the received incremental model, and then complete the dynamic topology update;

Step 3: the master system send the operation topology which is changed to the corresponding terminal, terminal and adjacent terminals make topological query, if it's consistent with the dynamic topology, program ends. If it is not consistent, reporting master system error, and GIS system will reidentify the topology model.

\section{Processing scheme for dynamic topology change of distribution line}

When the distribution line dynamic topology changes, that is, the local distribution network topology changes caused by the distribution line load transfer, fault isolation, non fault area after the restoration of power supply. From the point of view of dynamic topology change of distribution line, whether it is load transfer before fault, or fault isolation after the failure, non fault area recovery power supply, The essential feature is due to the sudden change of the switch status. Therefore, combined with the coloring function of distribution station system, and based on the Interaction mechanism of topology model of distributed FA terminal and main station system . Dynamic topology change algorithm is introduced according to the different working conditions. 
The adaptive algorithm for distribution line topology change caused by fault isolation after fault isolation is shown in figure 4.

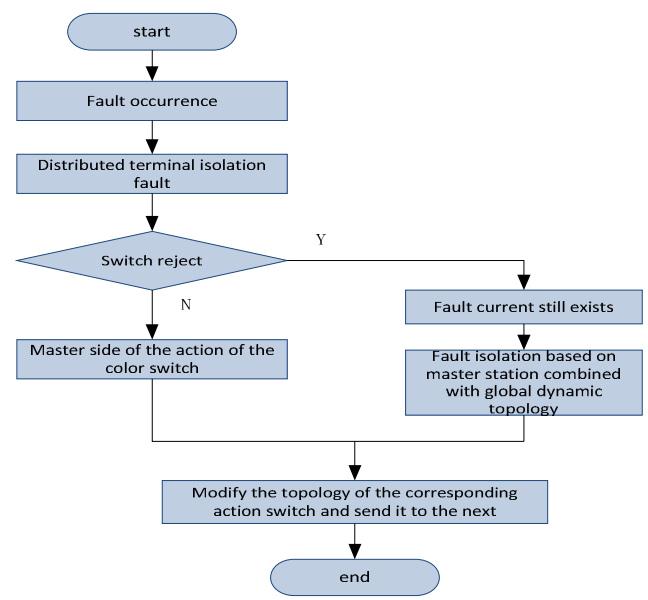

Fig. 4 Topology adaptation of power distribution lines for fault isolation

After the failure of the distribution line, Distributed FA enter the fault isolation procedures. Distribution automation terminal determine the occurrence of the fault point through the coordination and cooperation, then command the fault point on both sides of the adjacent switch off. If the switch refuses to move or location error appears, fault current still exists. The existence of fault current is detected after $200 \mathrm{~ms}$, the main station system start the fault isolation procedures. After the completion of the fault isolation, the main station system will update the area topology and the topology operation model will be carried out.

Load transfer before fault and non fault area recovery power supply, the essence of which is to change the corresponding load of the power supply path. So it merges into one kind of working condition. An adaptive algorithm for distribution line topology change caused by distribution line load transfer is shown in Figure 6.

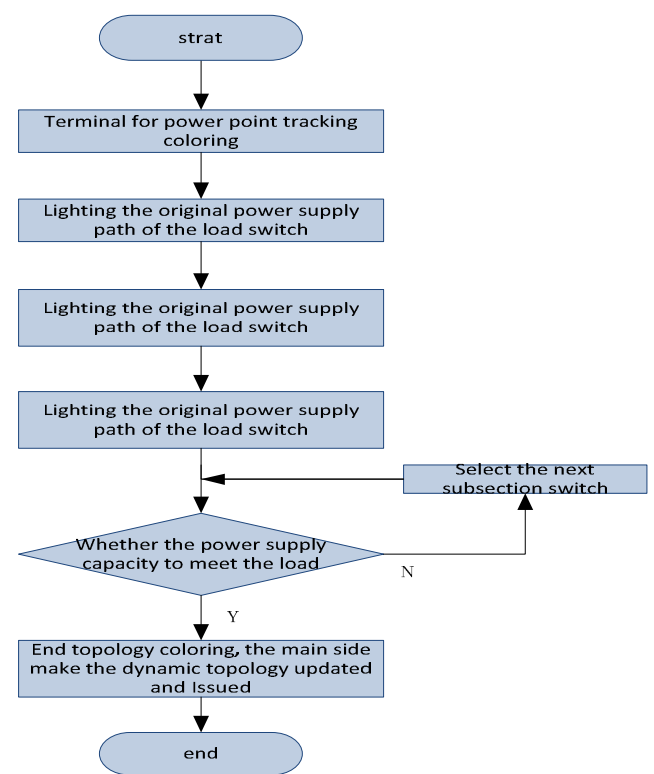

Fig. 5 Topology adaptation of power distribution lines for load transfer

After entering the load transfer program, the corresponding terminals make power point tracking coloring according to the storage topology operation model. In the monitoring interface of the main station system, the original power supply path of the load switch is lit. Then, the terminal automatically searches for all the contact switches that are close to the load switch, and interact with the main station system, all distribution lines that will be able to carry out the load will be displayed in different colors. At last, according to the color line, the power load capacity calculation is carried out automatically. If the power supply load capacity is not enough, entering the next line; If the power supply load capacity is enough, the status of the corresponding contact switch will be changed from 
the "point" to "hop", and ending topology coloring. The main station will down the dynamic topology updated.

\section{The test of intelligent terminal with network capacity of distribution line topology}

\section{Static model test}

In order to verify the effectiveness of the proposed scheme, The distribution line test system is established on the Nari static simulation system. The system can simulate the normal operation of distributed FA, fault processing, the interaction between distribution automation terminal and main station system based on the topology and adaptive process of distribution line static topology and dynamic topology. Master station system using OPEN5000, distribution circuit breakers' state can be controlled manually, each switch configuration a distribution terminal. The optical fiber Ethernet passive optical network (EPON) is used to achieve the transmission of information, and all the distribution terminals are connected to the peer to peer communication network. The test system of distribution line is shown in Figure 6.

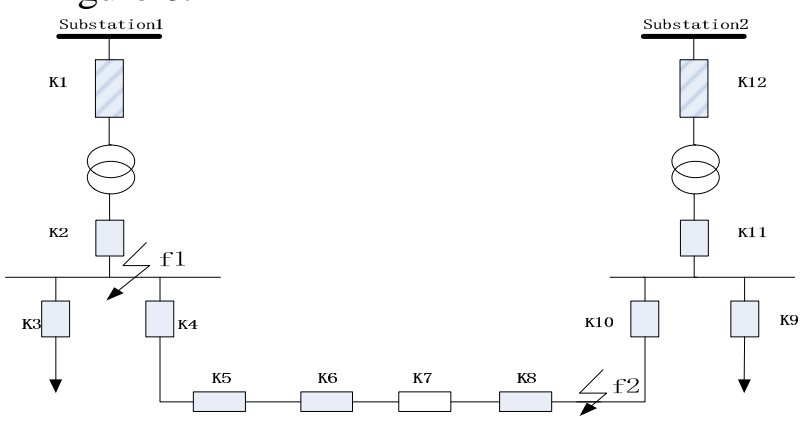

Fig. 6 Schematic diagram of test system for distribution line

In order to verify the effect of normal operation of distributed FA, fault processing, the interaction between distribution automation terminal and main station system based on the topology, the following experiments were carried out.

When the system is running normally, the contact switch K7 is in the state of split gate. Single phase to ground fault is provided in F1 and F2 points, as shown in figure. The distributed FA fault processing, the interaction between the master station and the terminal topology are recorded. The recorded contents include the time of fault isolation, the power supply time of the non fault area recovery, and the time for the terminal to receive the update operation topology of the main station after each operation.

When the fault occurs in the F1 position, the FTU Control circuit breaker, the distributed FA control K2, K4 to isolate the fault. After the completion of the fault isolation, the terminal receives the updated operation topology, and then the distributed FA control K1, the contact switch $\mathrm{K} 7$ to restore the non fault area power supply based on the fault information and the new operation topology. Then completes the terminal operation topology update again. The processing procedure of the F2 position is the same as the location of F1. Table 1 records the time of action of each stage. The operation topology receiving time includes the time of message transmission and the topology of the main station.

Table 1 The time of action of each stage of fault treatment

\begin{tabular}{lllll}
\hline $\begin{array}{l}\text { fault } \\
\text { point }\end{array}$ & $\begin{array}{l}\text { Fault } \\
\text { isolation } \\
\text { time } / \mathrm{ms}\end{array}$ & $\begin{array}{l}\text { Topology } \\
\text { receive } \\
\text { time } / \mathrm{ms}\end{array}$ & $\begin{array}{l}\text { Non fault } \\
\text { zone } \\
\text { recovery } \\
\text { time } / \mathrm{ms}\end{array}$ & $\begin{array}{l}\text { Topology } \\
\text { receive } \\
\text { time } / \mathrm{ms}\end{array}$ \\
\hline $\mathrm{f} 1$ & 261 & 310 & 282 & 308 \\
\hline $\mathrm{f} 2$ & 262 & 315 & 286 & 312 \\
\hline
\end{tabular}

The test results show that the distribution automation terminal and the main station system based on the topology of distribution lines can fully meet the requirements of distribution line fault isolation, 
non fault area to restore power supply, which can make fault processing time less than $1 \mathrm{~s}$, the topology of distribution line is completely updated less than 1.2s.

\section{Demonstration application}

In Nanjing Jiangbei District, the city of "O - type double - loop" distribution line test run. Through the deployment with the topology of distribution line networking capability of distribution automation terminal, to achieve fast fault isolation based on intelligent distributed FA, non fault area rapid restoration of power supply, and the realization of intelligent distributed FA network topology of ad hoc network technology.

The topology of the test circuit as shown in Figure 1, equipped with seven FTU, the two terminals of the circuit breaker, the system can update the operation of distribution circuit topology in time. Any failure in the line can be handled in $1 \mathrm{~s}$.

\section{Conclusions}

In this paper, technology of intelligent distribution terminal with the ability of ad hoc network for distribution line is proposed. Automatic updating of operating topology in distribution terminal is achieved through the interaction mechanism between the master station system and distribution automation terminal, and between terminals. The system has the advantages of rapid fault isolation and self healing, reducing the impact caused by fault area judgment and switch malfunction, and improving the operation reliability of distribution network. Static model test and demonstration application results show that, distribution automation terminal on distribution line can fully meet the functional requirements of distributed FA, and the line topology model installed in the distribution automation terminal can be updated rapidly and accurately. The method solves the problem that the topology model of the terminal can not be updated in the distributed FA application, and it has very important significance to promote the development of the distributed FA technology.

\section{Acknowledgements}

This work was financially supported by the "technology of intelligent distribution terminal with the ability of Ad hoc network for distribution line"

\section{References}

[1] HAN Guozheng, XU Bingyin, SUONAN Jiale, et al. Automation of Electric Power Systems, 2012, 36(18): 82-85.

[2] HAN Guozheng, XU Bingyin. Electric Power Automation Equipment, 2011, 31(2): 104-107.

[3] Han Guozheng, Xu Bingyin, Zhang Haitai etc.. Electric power Automation Equipment, 2011,31

(5): 99-102.

[4] Zhu ZhengYi, Xu Bingyin, Han Guozheng. Automation of Electric Power Systems,2015.39 (21): 144-149.

[5] Zhou Wenjun, Li Chunjian, Wang Liang. Power System Protection and Control, 2013, (17): 71-76.

[6] Xu Bingyin, Han Guozheng, SUONAN Jiale, et al. Power System Protection and Control, 2013, (2): 62-66.

[7] Tang Chenghong,Yang Zhihong,Song Bin, et al. Automation of Electric Power Systems,2015,39 (9): 101-106.

[8] CHEN Zhiwei, XU Bingyin, HAN Guozheng, et al. Electric Power Automation Equipment, 2013, 33(3): 136-140. 